\title{
OUTLET STRUT FRACTURE OF BJÖRK-SHILEY CONVEXO-CONCAVE VALVES: CAN VALVE-MANUFACTURING CHARACTERISTICS EXPLAIN THE RISK?
}

\author{
Rumana Z. Omar, $\mathrm{PhD}^{\mathrm{a}^{*}}$ \\ Linda S. Morton, BA Hons, RN ${ }^{\mathrm{a}}$ \\ Mairead Beirne, BA Hons ${ }^{\mathrm{a}}$ \\ William J. Blot, $\mathrm{PhD}^{\mathrm{b}}$ \\ Patricia V. Lawford, $\mathrm{PhD}^{\mathrm{c}}$ \\ Rodney Hose, $\mathrm{PhD}^{\mathrm{c}}$ \\ Kenneth M. Taylor, FRCS ${ }^{\text {a }}$
}

Background: Björk-Shiley $60^{\circ}$ convexo-concave prosthetic heart valves (Shiley, Inc, Irvine, Calif, a subsidiary of Pfizer, Inc) continue to be a concern for approximately 35,000 nonexplanted patients worldwide, with approximately 600 events reported to the manufacturer to date. Fractures of the outlet struts of the valves began to appear in the early 1980s and have continued to the present, but their causes are only partially understood.

Methods: A matched case-control study was conducted evaluating manufacturing records for 52 valves with outlet strut fractures and 248 control subjects matched for age at implantation, valve size, and valve position.

Results: In addition to the risk factors recognized as determinants of outlet strut fracture, the United Kingdom case-control study has observed 7- to 9fold increased risk with performance of multiple hook deflection tests. This test was performed more than once, usually after rework on the valve. Six valves in this study underwent multiple hook deflection tests, of which 4 experienced an outlet strut fracture. Cracks and further rework were noted for these valves. Significant associations were also observed between outlet strut fracture and disc-to-strut gap measurements taken before the attachment of the sewing ring.

Conclusions: It is our view that a combination of factors related to valve design, manufacturing process, and patient characteristics are responsible for outlet strut fractures of Björk-Shiley convexo-concave valves. Multiple hook deflection tests have emerged as a potential new risk factor for outlet strut fracture in both The Netherlands and the United Kingdom. This factor appears to be correlated with the presence of other abnormalities. A further study is needed to investigate the factors correlated with multiple hook deflection tests. On confirmation of risk, the presence of multiple hook deflection tests may be added to equations, quantifying the risk of outlet strut fracture for comparison against risk of mortality and serious morbidity from explant operations. (J Thorac Cardiovasc Surg 2001;121:1143-9)
From the Departments of Cardiac Surgery and Epidemiology and Public Health, Imperial College of Science, Technology and Medicine, Hammersmith Hospital, London, United Kingdoma, the International Epidemiology Institute, Rockville, $\mathrm{Md}^{\mathrm{b}}$; and the Department of Medical Physics and Clinical Engineering, The University of Sheffield, Royal Hallamshire Hospital, Sheffield, United Kingdom. ${ }^{c}$

Supported by the Trustees for the Bowling Pfizer Heart Valve Settlement Funds.

*Present address: Department of Statistical Science, University College London, Gower Street, London WC1E 6BT, United Kingdom.

Copyright (C) 2001 by The American Association for Thoracic Surgery

0022-5223/2001 $\$ 35.00+0 \quad \mathbf{1 2 / 1 / 1 1 3 9 3 7}$

doi:10.1067/mtc.2001.113937
0 utlet strut fractures (OSFs) of Björk-Shiley convexo-concave (BSCC) valves (Shiley, Inc, Irvine, Calif, a subsidiary of Pfizer, Inc) continue to be a concern for approximately 35,000 nonexplanted patients worldwide. Although reports of OSF events were highest in the 1980s, particularly among large mitral valves, it is important to note that OSF events are still occurring, and as of September 1999, over 600 events have been reported to the manufacturer (Trustees for the Bowling Pfizer Heart Valve Settlement Funds, personal communication). Additionally, an underreporting of $10 \%$ to $25 \%$ for OSF events has been suggested in various studies. ${ }^{1-4}$ An increased risk of OSF for $23-\mathrm{mm}$ 
aortic valves has emerged more recently in the United Kingdom, with the last OSF reported in September 2000.

There has been much debate as to whether the problem of OSF lies with the intrinsic valve design, specific characteristics of the valve-manufacturing process, or patient characteristics. Previous research has identified age at implantation and body surface area as key patient factors associated with the risk of OSF. ${ }^{1,2,5}$ No dominant clinical factor of risk has been identified. ${ }^{1}$ Of the valve characteristics investigated, large valve size, mitral position, and a $70^{\circ}$ opening angle are thought to be associated with risk of OSF. ${ }^{2-4,6}$ With respect to the manufacturing process, valves manufactured after 1981 in batches with high fracture rates and welded by specific groups of employees were reported to be at a high risk of OSF. 1,6-8 The current guidelines ${ }^{9}$ for the patients and clinicians concerning explant operations for BSCC valves include these manufacturing and valve characteristics and current age in predicting the risk of OSF. However, these factors do not fully explain the risk with OSF events occurring outside these parameters. The development of guidelines for explant operations is a continuous process and is updated on the basis of ongoing research. It is therefore important to examine various aspects of the valve-manufacturing process in detail to identify other potential risk factors. Two studies have conducted indepth investigations on various manufacturing characteristics of BSCC valves, but they differ in terms of factors investigated and their findings. ${ }^{6,7}$

This article describes a case-control study nested within the cohort study of patients with BSCC valves in the United Kingdom. ${ }^{1}$ The objective of this study was to examine the risk of OSF in relation to combined characteristics of the manufacturing process investigated by Walker, ${ }^{6}$ Kallewaard, ${ }^{7}$ and their colleagues. Additionally, information on a number of other manufacturing characteristics considered to be potential risk factors for OSF were examined.

\section{Methods}

Selection of cases and controls. A nested case-control study was conducted in which OSF events with supporting documentation available up to the last follow-up date (July 31,1997 ) of the UK cohort study were eligible to be cases. ${ }^{1}$ The case-control study includes 52 of the 56 OSF events reported in this cohort study. Two of the OSF events occurring within the last follow-up date of the cohort had documented evidence available at a later period. Manufacturing records were not available for 2 further OSF events. Five control subjects were selected for each case from the study cohort matched on age at implantation, valve position, and size. However, 10 of the selected control subjects had to be dropped from the study because of information received sub- sequently, which made them ineligible for inclusion. It was only possible to obtain 3 matching control subjects within the study cohort for one 23-mm aortic OSF. The UK case-control study thus comprises 52 cases with OSF events and 248 matched control subjects, including at least 3 matching control subjects for each case. Manufacturing records were obtained from the manufacturer.

Data abstraction. The characteristics of the manufacturing process of BSCC valves and related documentation have been described extensively elsewhere ${ }^{6,7}$ and are only discussed briefly in this article. A simplified version of the manufacturing process is presented in Fig 1 to assist the reader. The main manufacturing characteristics, which were examined for association with the risk of OSF in the UK case-control study, are presented in Table I. The selection of factors was based on a review of the manufacturer's engineering specifications, consultation with a metallurgist, and evaluation of aspects of the manufacturing process investigated by the studies of Walker, ${ }^{6}$ Kallewaard, ${ }^{7}$ and their colleagues.

Variables 1 to 5 in Table I pertain to information on the outlet strut assembly production time, the manufacturing period, the size of the production batch (lot size), and the percentage of valves rejected in a batch during quality-control procedures. These factors were considered to be a measure of the quality of the batch of valves in which they were produced.

The remaining variables describe tasks performed on the individual valve during the manufacturing process. Some tasks, such as disc fit and assembly and disc pull, require manipulation of the actual outlet strut of the valve. Factors describing the extent of rework done on the valves included the number of discs fitted to the valve, rewelding of deficient welds, and removal of excess weld material by polishing (relieve weld).

The flexibility tests were performed on the valves' outlet strut after the welding process to ascertain the amount of deflection of the strut when subjected to a specific force or load. Two types of flexibility tests known as the hook deflection test (HDT) and load deflection test (LDT) were carried out, depending on when the valve was manufactured. The HDT was replaced in February 1982 by the LDT; however, valves that had production times crossing over from before February 1982 to a later period and those that were recalled could have undergone both types of tests. In performing an HDT, a 7-kg weight was suspended from the outlet hook for $21-$ to $29-\mathrm{mm}$ valves and a $5-\mathrm{kg}$ weight for $19-\mathrm{mm}$ valves. After August 1981 and until its withdrawal, this test was allowed to be performed more than once, usually after rework on the valve. For an LDT, a 5-kg load was gradually placed on the outlet strut, unloaded, and then reapplied.

The layout and information recorded on the manufacturing documents changed over time, resulting in several different versions, and were categorized by Kallewaard and colleagues $^{7}$ to reflect the time period in which they were used. The baggy card is a manufacturing document that records information on standard assembly tasks and reworking, and version 4 was most commonly used. This version pertains to the manufacturing period from 1981-1982, which was observed to be a risk factor for OSF. ${ }^{8,9}$ 
Table I. Results from univariate regression analyses on the basis of valves

\begin{tabular}{|c|c|c|c|c|}
\hline Manufacturing characteristics & & Patients/control subjects & Odds ratio & $95 \% C I$ \\
\hline \multicolumn{5}{|l|}{ Batch level documents } \\
\hline \multirow[t]{2}{*}{ Size of lot to be assembled* } & $<175$ valves & $25 / 117$ & & \\
\hline & $\geq 175$ valves & $27 / 131$ & 1.0 & $0.5-1.8$ \\
\hline \multirow[t]{3}{*}{ Production time per valve ${ }^{\dagger}$} & $>0.16 \mathrm{~d}$ (baseline) & $14 / 85$ & & \\
\hline & $0.12-0.16 \mathrm{~d}$ & $27 / 89$ & 2.1 & $1.0-4.6$ \\
\hline & $<0.12 \mathrm{~d}$ & $11 / 74$ & 1.0 & $0.4-2.4$ \\
\hline \multirow[t]{2}{*}{ Manufacturing date } & Before 1981 (baseline) & $4 / 43$ & & \\
\hline & $1981-1984$ & $48 / 205$ & 3.8 & $1.0-13.7$ \\
\hline SFR & & $52 / 248$ & 1.3 & $1.1-1.5$ \\
\hline Percentage of valves rejected & & $51 / 245$ & 1.0 & $0.9-1.0$ \\
\hline \multicolumn{5}{|l|}{ Individual valve documents } \\
\hline \multirow[t]{2}{*}{ Baggy version } & Other versions (baseline) & $14 / 67$ & & \\
\hline & Version 4 & $38 / 181$ & 1.0 & $0.5-2.3$ \\
\hline \multirow[t]{2}{*}{ Disc fit recorded more than once } & No (baseline) & $38 / 207$ & & \\
\hline & Yes & $14 / 41$ & 1.8 & $0.9-3.6$ \\
\hline \multirow[t]{2}{*}{ Final polish recorded more than once } & No (baseline) & $40 / 199$ & & \\
\hline & Yes & $12 / 42$ & 1.4 & $0.7-3.0$ \\
\hline \multirow[t]{3}{*}{ No. of HDTs performed } & None (baseline) & $34 / 158$ & & \\
\hline & 1 & $14 / 88$ & 0.7 & $0.3-1.5$ \\
\hline & $\geq 2$ & $4 / 2$ & 8.1 & $1.4-45.8$ \\
\hline \multirow[t]{3}{*}{ Number of LDTs performed } & None (baseline) & $16 / 106$ & & \\
\hline & 1 & $34 / 125$ & 2.8 & $1.5-6.8$ \\
\hline & $\geq 2$ & $2 / 17$ & 1.2 & $0.3-6.4$ \\
\hline \multirow[t]{2}{*}{ Disc assembly recorded more than once } & No (baseline) & $33 / 175$ & & \\
\hline & Yes & $19 / 73$ & 1.4 & $0.7-2.7$ \\
\hline \multirow[t]{2}{*}{ Relieve weld recorded more than once } & No (baseline) & $47 / 230$ & & \\
\hline & Yes & $5 / 23$ & 1.4 & $0.5-4.1$ \\
\hline \multirow[t]{2}{*}{ Crack or weld noted } & No (baseline) & $44 / 223$ & & \\
\hline & Yes & $8 / 25$ & 1.7 & $0.7-4.1$ \\
\hline \multirow[t]{2}{*}{ Disc pull noted } & No (baseline) & $49 / 223$ & & \\
\hline & Yes & $3 / 25$ & 0.6 & $0.2-1.9$ \\
\hline \multirow[t]{2}{*}{ More than one disc used for this valve } & No (baseline) & & & \\
\hline & Yes & & 1.5 & $0.4-5.5$ \\
\hline \multirow[t]{2}{*}{ Red mark noted } & No (baseline) & $47 / 204$ & & \\
\hline & Yes & $5 / 44$ & 0.5 & $0.2-1.3$ \\
\hline \multirow[t]{4}{*}{ Disc-to-strut measurement ${ }^{\ddagger}$ (inch) } & $<0.012$ (baseline) & $7 / 75$ & & \\
\hline & 0.012 & $18 / 86$ & 2.6 & $1.0-6.9$ \\
\hline & 0.013 & $7 / 42$ & 2.4 & $0.7-7.8$ \\
\hline & $>0.013$ & $20 / 40$ & 8.8 & $2.9-26.8$ \\
\hline \multirow[t]{4}{*}{ Disc-to-strut measurement ${ }^{\S}$ (inch) } & $<0.012$ (baseline) & $12 / 86$ & & \\
\hline & 0.012 & $14 / 79$ & 1.4 & $0.6-3.2$ \\
\hline & 0.013 & $10 / 33$ & 2.5 & $0.9-6.6$ \\
\hline & $>0.013$ & $16 / 49$ & 2.9 & $1.1-7.3$ \\
\hline \multirow[t]{2}{*}{ Phantom welder present } & No (baseline) & $50 / 242$ & & \\
\hline & Yes & $2 / 6$ & 1.6 & $0.3-8.0$ \\
\hline
\end{tabular}

Number of patients and control subjects for each variable may not always add up to 52 and 248 , respectively, because of missing observations.

*Based on median lot size of the Dutch study for comparability.

†Based on tertiles.

†Taken before sewing ring placement.

$\S$ Taken after sewing ring placement

A red tag often used to mark reworked valves was examined as a surrogate for rework status. In addition, 2 other factors were investigated, the disc-to-strut gap (DSG) and the presence of a phantom welder. The DSG measures the maximum clearance from the disc to the outlet strut at any opening angle of the valve. It was thought that this could be a determinant for excess movement of the disc within the struts. Two sets of measurements were available for DSG taken before and after the sewing ring was fit- ted to the valve. The phantom welder refers to the employee number of an individual who was recorded on the manufacturing records as having performed rewelding on specific valves but was not employed by the manufacturer when the valves were allegedly rewelded. ${ }^{7}$ Finally, shop-order fracture rate (SFR), which was defined as the percentage of other valves in the same batch for which an OSF event had been reported to the manufacturer, and manufacturing period were examined as risk factors. 




Fig 1. A simplified description of manufacturing procedures of BSCC heart valves. *Assessment of disc size, disc not fitted. \#Regurgitation tests completed. When valves fail at specific points in the manufacturing process, they are reworked and will be returned to the failed test point or to an earlier process. **Valves manufactured before February 1982 underwent HDT, and those manufactured later underwent LDT; some underwent both.

All documents were double data abstracted and entered. Methods of data abstraction and coding were specifically designed to be similar to those used in the investigation of Kallewaard and colleagues. ${ }^{7}$ A conditional logistic regression analysis was performed to examine the potential risk factors in relation to OSF. All statistical analyses were carried out by using the statistical software STATA (Stata Corporation, College Station, Tex). Both univariate and multiple-regression analyses were performed. Variables that had been identified as risk factors in previous studies and also those for which $P$ values were .2 or less in the univariate analysis were included in the multiple-regression analyses. A backward elimination procedure ${ }^{10}$ was used to select the final model.

\section{Results}

There were 40 mitral OSF cases with 193 control subjects and 12 valves with aortic OSFs with 55 control subjects in the study. Table I presents the results from the univariate regression analyses. Of all the variables examined, manufacturing date, SFR, performance of a multiple HDT, performance of an LDT, and DSG measurements were found to have statistically significant associations with the risk of OSF. There was a decrease in the risk of OSF when an HDT was performed only once. The excess risk associated with LDT was only significant when performed once. It is somewhat difficult to disentangle the influence of these flexibility tests from one another because 21 valves in this study had undergone both HDT and LDT. Four of the 6 valves that had undergone multiple HDTs had also undergone a single LDT. However, none of the valves underwent both types of flexibility tests more than once. Because of the small numbers, it was not possi- 
Table II. Results from multiple regression analyses on the basis of all valves

\begin{tabular}{|c|c|c|c|c|c|c|}
\hline \multirow[b]{2}{*}{ Variables } & \multicolumn{2}{|c|}{ Model 1} & \multicolumn{2}{|c|}{$\begin{array}{c}\text { Model } 2 \\
\text { (including first } \\
\text { DSG measurement) }\end{array}$} & \multicolumn{2}{|c|}{$\begin{array}{c}\text { Model } 3 \\
\text { (including second } \\
\text { DSG measurements) }\end{array}$} \\
\hline & $O R$ & $95 \% C I$ & $O R$ & $95 \% C I$ & $O R$ & $95 \% \mathrm{Cl}$ \\
\hline Size of lot assembled $\geq 175$ valves ${ }^{*}$ & 1.0 & $0.5-1.9$ & & & & \\
\hline More than 1 disc used for this valve ${ }^{\dagger}$ & 0.8 & $0.1-5.2$ & & & & \\
\hline \multicolumn{7}{|l|}{ Number of HDTs } \\
\hline 1 & 0.5 & $0.2-1.3$ & 0.8 & $0.3-2.1$ & 0.5 & $0.2-1.4$ \\
\hline$\geq 2$ & 7.5 & $1.1-50.6$ & 8.9 & $1.3-61.4$ & 7.0 & $1.1-45.5$ \\
\hline Percentage of SFR & 1.3 & $1.1-1.6$ & 1.3 & $1.1-1.6$ & 1.3 & $1.1-1.5$ \\
\hline \multicolumn{7}{|l|}{ DSG (inch) $)^{\S}$} \\
\hline 0.012 & & & 1.7 & $0.6-4.8$ & 0.8 & $0.3-2.2$ \\
\hline 0.013 & & & 1.6 & $0.5-5.8$ & 1.6 & $0.6-4.6$ \\
\hline$>0.013$ & & & 7.1 & $2.2-23.1$ & 1.7 & $0.6-4.7$ \\
\hline
\end{tabular}

$O R$, Odds ratio.

*Baseline < 175 valves.

'Baseline: one disc used.

No HDTs performed.

$\S$ Baseline: $<0.012$ inch.

Table III. Some manufacturing characteristics of valves that underwent multiple HDTs

\begin{tabular}{|c|c|c|c|c|c|c|c|c|c|c|}
\hline Observations & OSF & $\begin{array}{c}\text { Valve } \\
\text { position }\end{array}$ & $\begin{array}{l}\text { Valve } \\
\text { size }\end{array}$ & $\begin{array}{c}\text { No. of } \\
\text { times disc } \\
\text { fit recorded }\end{array}$ & $\begin{array}{c}\text { LDTs } \\
\text { performed }\end{array}$ & $\begin{array}{l}\text { Crack/ } \\
\text { reweld } \\
\text { noted }\end{array}$ & $\begin{array}{c}\text { Phantom } \\
\text { welder } \\
\text { noted }\end{array}$ & $\begin{array}{c}\text { No. of times } \\
\text { final polish } \\
\text { recorded }\end{array}$ & $\begin{array}{l}\text { No. of disc } \\
\text { assemblies }\end{array}$ & $\begin{array}{l}\text { Disc } \\
\text { pull }\end{array}$ \\
\hline 1 & Yes & Mitral & 33 & 3 & Yes & Yes & Yes & 3 & 2 & Yes \\
\hline 2 & Yes & Mitral & 31 & 4 & Yes & No & No & 2 & 9 & No \\
\hline 3 & Yes & Mitral & 33 & 1 & Yes & No & Yes & 3 & 6 & No \\
\hline 4 & Yes & Aortic & 23 & 1 & No & Yes & Yes & 2 & 4 & Yes \\
\hline 5 & No & Mitral & 29 & 1 & Yes & Yes & Yes & 2 & 2 & Yes \\
\hline 6 & No & Mitral & 27 & 3 & No & Yes & No & 2 & 2 & Yes \\
\hline
\end{tabular}

ble to conduct a further analysis investigating the risk of OSF by grouping the valves according to whether they had undergone only one type of flexibility test (single and multiple) or a combination of both.

Results from the multiple-regression analyses are presented in Table II. Initially, an analysis was conducted, including only manufacturing characteristics identified as the main risk factors in the study by Kallewaard and colleagues (model 1). ${ }^{7}$ Age at implant, which has been used as a matching factor in the study design, was not included in this model. SFR and performance of a multiple HDT were observed to be significantly related to the risk of OSF, but lot size and the number of discs fitted did not show any association. A second multiple-regression analysis was performed to identify significant risk factors for OSF on the basis of the UK data (models 2 and 3). Manufacturing period and LDT were not significant in these analyses, whereas associations observed with SFR and multiple HDTs remained significant. There was an increased risk in
OSF for DSG measurements of greater than $0.013 \mathrm{inch}$, but the association was only significant for measurements taken after the attachment of the sewing ring.

Similar results were obtained when the analyses were restricted to large mitral valves only.

Six of the 20 cases that had a DSG of greater than 0.013 inch when measured before the attachment of the sewing ring moved to a lower measurement category after the attachment. A similar pattern was observed for only 7 of the 40 control valves with a first DSG measurement of greater than 0.013 inch. This resulted in weakening the association with the second set of DSG measurements, but the reason behind this differential reduction is not clear.

Further investigation was made on the characteristics of the 6 valves that underwent multiple HDTs. Some of the manufacturing characteristics of these valves are presented in Table III. Four of the valves had a crack or reweld documented, and 2 experienced an OSF. Three of the 4 valves that had also undergone an LDT had 
fractured. There was one OSF valve that had a disc fitted 4 times, and 9 disc assembly procedures were recorded. The median time taken for the valves undergoing multiple HDTs to fracture from the time of implant (median, 5 years; range, 0.7-9.6 years) was similar to that of the other OSF valves (median, 5 years; range, 0.6-15.7 years) not undergoing multiple HDTs. DSG measurements did not show any association either with multiple HDTs or with rework.

Investigations were also made to see whether there were any differences in the SFR, the number of HDTs performed, and DSG measurements by manufacturing period. The SFR and DSG measurements were smaller before 1981. The SFR was highest during January 1, 1981, and June 1982, and all 6 valves that underwent multiple HDTs were manufactured during that period.

\section{Discussion}

OSFs in BSCC valves continue to be a matter of concern both for patients with BSCC valves and for clinicians. The UK cohort of BSCC patients has a longer and more complete follow-up than that reported in other published studies. ${ }^{1}$ The crude OSF rate in the United Kingdom was estimated to be $0.17 \%$ per year, the highest among all monitored populations. To date, 61 OSF events have been reported in the United Kingdom, with 51 resulting in the death of the patient. Eight OSF events have occurred within the last 5 years, which include 5 aortic OSFs.

A changing pattern of OSF rates has been observed in the UK cohort, with mitral OSF rates reducing over time but without the same pattern for aortic OSF rates. An excess risk has particularly been observed in 23$\mathrm{mm}$ aortic valves. Aortic OSFs usually result in instantaneous death and consequently may be underreported to a greater extent. The excess in 23-mm aortic valves has been seen in Sweden, ${ }^{3}$ where the postmortem rate is high, but not in The Netherlands ${ }^{4}$ or the United States. ${ }^{5}$ Accuracy of detecting OSF events in the United Kingdom is thought to be high. All patients are tracked by the Office of National Statistics and the Central Services Agency for information on their vital status and forwarding of death certificates for systematic review. The postmortem rate in the United Kingdom is $30 \%$ for all deaths and $49 \%$ for sudden deaths. A larger number of mitral valves than aortic valves have been electively explanted in the United Kingdom because the former were considered to be at greater risk. This may partly explain the changing OSF patterns in the United Kingdom.

The UK case-control study thus differs from other studies with respect to a broader spectrum of valves included and also a wider range of manufacturing characteristics investigated. Kallewaard and colleagues ${ }^{7}$ only considered large mitral valves, including $26 \mathrm{OSF}$ events (half the number included in the UK study), and identified performance of multiple HDTs, number of discs fitted to the valve, and lot size as risk factors. The finding of multiple HDTs has been confirmed in the United Kingdom, with a sizeable increase in the risk of OSF associated with this factor (7- to 9-fold risk), but associations with the other factors were not observed. The application of a large weight to the outlet strut during the HDT may have been excessive, although only multiple HDTs were associated with increased risk of OSF, and a reapplication may cause permanent deformation. Of the 4 OSF valves that had undergone multiple HDTs in the UK case-control study, some also had a crack or reweld documented and had undergone an additional flexibility test (LDT). After adjusting for these factors, multiple HDTs remained significant; however, it is not possible to conclude definitely that multiple HDTs on their own are causal risk factors for OSF. Nevertheless, it is clear that multiple HDTs are a marker for excess risk.

Walker and colleagues ${ }^{6}$ did not investigate the number of times the valves underwent an HDT or LDT but reported statistically nonsignificant excess risk with flexible struts. This finding could not be investigated in the United Kingdom because deflection measurements for valves that underwent HDTs and had results recorded only included 18 of the cases.

A high risk of OSF was observed with large DSG measurements but was only significant for the first set of measurements. A large gap could be indicative of the fact that the outlet strut is a long way forward from the edge of the disc, which could predispose the disc on overrotation to impact the strut above the weld area. It could also be an indication of incorrect seating of the disc on the inlet strut. However, it is difficult to draw definite conclusions regarding the DSG measurements. Excess risk was associated with gaps of greater than 0.013 inch for both sets of measurements but was only significant for the first set. The measurements were taken before and after the attachment of the sewing ring by different observers. The differences may be attributed to one or both of these factors. One could speculate that the measurements taken after the attachment of the sewing ring are more relevant because these measurements were taken before the valves were dispatched for implantation. Walker and colleagues ${ }^{6}$ found a nonsignificant protective effect for DSG measurements of less than 0.010 inch; however, they did not mention whether the results were based on the first 
or the second set of measurements. Kallewaard and colleagues ${ }^{7}$ did not report findings on this factor.

Body surface area has been identified as a risk factor for OSF ${ }^{1,5}$ The association with body surface area could not be investigated when HDT was included in the model because information on body surface area was not available in 2 of the 4 valves undergoing multiple HDTs that had experienced an OSF. However, body surface area remained significant after adjusting for SFR, manufacturing period, and DSG measurements.

Wieting and colleagues ${ }^{11}$ suggest a plausible combination of valve and patient factors as prerequisites for valve failure. In particular, they indicate that a mechanical load associated with an overrotation of the disc on closing could cause impact of the disc with the outlet strut. This, combined with patient physiology and valve geometric factors, is likely to be responsible for the observed fractures. On over-rotation, impacts at the tip of the strut or above the weld base may cause failure. Changes to the inspection process after April 1984 made certain that the former condition could not occur on a valve passing the inspection tests. These changes ensured correct alignment of the disc against the inlet strut in the closed position so that should the disc overrotate there would be no possibility of impact on the outlet strut leg in the area above the weld. No OSF events have been reported worldwide in valves manufactured after this period. Within the case-control study, it was therefore not possible to ascertain whether the DSG measurements had changed after the introduction of the tests in early 1984 .

It is our view that a combination of factors related to patients, design of the valves, and the manufacturing process are responsible for OSF of BSCC valves. Multiple HDTs have emerged in both The Netherlands and the United Kingdom as a potential new risk factor for OSF, and the estimated risk associated with it is one of the largest compared with other previously identified risk factors. To prepare guidelines to enable clinicians and patients to make decisions for explant operations, it is important to use risk factors that have been validated across studies and for which information can easily be obtained. A further study is needed to explore the factors correlated with multiple HDTs. On confirmation as a risk factor, the presence of multiple HDTs may be added to the models used to predict OSF risk, thus influencing decisions regarding the eligibility of patients for explant operations.
Cecile Beckers provided training for data abstraction, and Cees Haaring set up the database used for data entry. Debbie Halliday helped in implementing the abstraction process of the data. Becky Webb and Tom Allum helped with the data abstraction. Shahed Murad read through the manuscript and provided useful comments. We thank Sir Donald Acheson for his advice and support.

Received for publication Aug 30, 2000; revisions requested Oct 13, 2000; revisions received Dec 11, 2000; accepted for publication Dec 27, 2000.

Address for reprints: Professor K. M. Taylor, Department of Cardiothoracic Surgery, Imperial College of Science, Technology, and Medicine, Hammersmith Hospital, Du Cane Rd, London W12 ONN, United Kingdom.

\section{REFERENCES}

1. Omar RZ, Morton L, Halliday D, Danns E, Beirne M, Blot WJ, et al. Outlet strut fracture of Björk-Shiley Convexo Concave heart valves-The UK cohort study. Report for the Trustees for the Bowling-Pfizer Heart Valve Settlement Funds. Cincinnati, Ohio: Bowling-Pfizer Heart Valve Settlement Funds; 2000.

2. Van der Graaf Y, De Waard F, Van Herwerden LA, Defauw J. Risk of strut fracture of Björk-Shiley valves. Lancet 1992;339:257-61.

3. Lindblom D, Rodriguez L, Björk VO. Mechanical failure of the Björk-Shiley valve. J Thorac Cardiovasc Surg 1986;92:894-907.

4. Kallewaard M, Algra A, Defauw J, Grobbee D, Van der Graaf Y. Likelihood of underreporting of outlet strut fracture from examination of the Dutch Björk-Shiley CC cohort. Am J Cardiol 1998;82:768-73.

5. Walker AM, Funch DP, Sulsky SI, Dreyer NA. Patient factors associated with strut fracture in Björk-Shiley 60 degree convexoconcave heart valves. Circulation 1995;92:3235-9.

6. Walker AM, Funch DP, Sulsky SI, Dreyer NA. Manufacturing characteristics associated with strut fracture in Björk-Shiley 60 degree convexo-concave heart valves. J Heart Valve Dis 1995;4:640-8

7. Kallewaard M, Algra A, Defauw J, Van der Graaf. Which manufacturing characteristics are predictors of outlet strut fracture in large sixty-degree Björk-Shiley convexo-concave mitral valves? J Thorac Cardiovasc Surg 1999;117:766-74.

8. Hiratzka LF, Kouchoukos NT, Grunkemeier GL, Miller DC, Scully HE, Wechsler AS. Outlet strut fracture of the Björk-Shiley $60^{\circ} \mathrm{CC}$ convexo-concave valve: current information and recommendations for patient care. J Am Coll Cardiol 1988;11:1130-7.

9. Important updated information for physicians about patients with Björk-Shiley Convexo-Concave Heart Valves. Trustees for the Bowling-Pfizer Heart Valve Settlement Funds. Cincinnati, Ohio: Bowling-Pfizer Heart Valve Settlement Funds; 2000.

10. Altman D. Practical statistics in medical research. London: Chapman and Hall; 1991.

11. Wieting DW, Eberhardt, Reul H, Eugene MB, Schreck SG, Chandler JG. Strut fracture mechanisms of the Björk-Shiley Convexo-Concave Heart Valve. J Heart Valve Dis 1999;8:206-17. 